\section{Nauplius}

The Journal of The Brazilian Crustacean Society

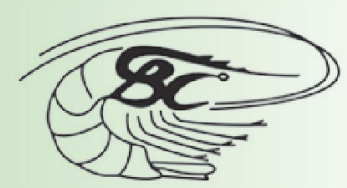

e-ISSN 2358-2936

www.scielo.br/nau www.crustacea.org.br

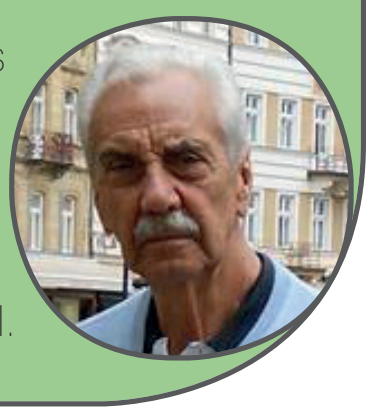

\title{
An integrative approach: using field and laboratory data to characterize shell utilization and selection pattern by the hermit crab Pagurus criniticornis (Paguridae) from Anchieta Island, Brazil
}

\author{
Fernando L. Mantelatto ${ }^{1}$, Fabíola C. R. Faria ${ }^{1,2}$, Renata Biagi
} and Andrea L. Meireles ${ }^{1}$
1 Laboratório de Bioecologia e Sistemática de Crustáceos (LBSC), Departamento de Biologia, Faculdade de Filosofia, Ciências e Letras de Ribeirão Preto (FFCLRP), Universidade de São Paulo (USP). Av. Bandeirantes, 3900. 14040-901 Ribeirão Preto, São Paulo, Brazil.
2 Núcleo Integrado de Biotecnologia, Universidade de Mogi das Cruzes. Av. Dr. Cândido Xavier de Almeida e Sousa, 200. 08780-911 Mogi das Cruzes, São Paulo, Brazil.

ZOOBANK: http://zoobank.org/urn:Isid:zoobank.org:pub:FFF42D8C-0693-4595B16E-6CBE240A738E

\author{
CORRESPONDING AUTHOR \\ Fernando L. Mantelatto \\ flmantel@usp.br \\ SUBMITTED 13 January 2016 \\ ACCEPTED 11 May 2016 \\ PUBLISHED 9 September 2016 \\ Guest Editors \\ Maria Lúcia Negreiros-Fransozo \\ and Adilson Fransozo
}

DOI 10.1590/2358-2936e201601 1

\section{AвsTRACT}

The aim of this study was to characterize the pattern of gastropod shell occupation in the field and selection of shell size and type under laboratory conditions by the hermit crab Pagurus criniticornis (Dana, 1852), inhabiting the infralittoral area of Anchieta Island, São Paulo, Brazil. Hermit crabs were obtained monthly during 1999 by SCUBA diving. For experiments under laboratory conditions, samplings were performed in 2002. The hermit crabs occupied 16 species of gastropods shells. Cerithium atratum (Born, 1778) was the most occupied shell (89.31\%), followed by Morula nodulosa (4.73\%) (Adams, 1845). No difference was observed in the pattern of occupation between males and females. The equations that best demonstrated the relationship between hermit crabs and their shells were those that involved Shell Wet Weight (SWW) and Shell Internal Volume (SIV). The laboratory experiments were in accordance to the pattern of occupation observed in the field; the mean value of SAI (Shell Adequacy Index) recorded to the population studied was 1.13 with a trend to increase this value in the last size classes. The results obtained corroborate with the hypothesis of the 
occupation process of shells governed not only by availability of shells, but also by its architecture. In addition, the shell stock in the area is one another important condition related to the exhibited pattern of shell occupation by $P$. criniticornis, and allows the stable coexistence among the island assemblage. The pattern of occupation observed promotes a high reproductive profile for the population studied, maximizing the populational growth.

\section{KEY WORDS}

Anomura, assemblage, Paguroidea, Pagurus, shell occupation.

\section{INTRODUCTION}

Pagurus criniticornis (Dana, 1852) is a tropical small species with geographic distribution restricted to Western Atlantic (Melo, 1999) and constitutes an abundant population in the hermit crab community of infralittoral areas of São Paulo coast (Meireles et al., 2012). Its records include some areas as Anchieta Island, where it has been reported together with other five coexisting species: Calcinus tibicen (Herbst, 1971), Pseudopaguristes calliopsis (Forest and Saint Laurent, 1968), Paguristes erythrops Holthuis, 1959, Paguristes tortugae Schmitt, 1933 and Pagurus brevidactylus (Stimpson, 1859) (Mantelatto and Garcia, 2002).

In natural populations, the pattern of occupation of gastropod shells observed by hermit crabs is dependent of many factors such as the local availability of different species of gastropods, the presence of sympatric species or by the hermit crabs' preference to certain species of gastropods shells (Bertness, 1981a; Dominciano et al., 2009). However, in complex communities compounded by more than two coexistent species, the explanations regarding the mechanisms responsible by the shell choice and/or shell preference remain unclear and are not satisfactory. The reasons, in most cases, are due to the absence of complete methods of evaluation, including natural and laboratory observations and the absence of knowledge regarding the resource (live gastropods and empty shells).

The objective of the present study was to determine the pattern of occupation and selection of gastropod shells by $P$. criniticornis population inhabiting the infralittoral of Anchieta Island, São Paulo, Brazil. We characterized morphometric relationships and the pattern of selection for shell size and type under laboratory conditions, in order to provide subsidy to elucidate questions about the dynamics of the hermit crabs' community on this island and investigate the hypothesis of occupation conducted by availability resource (shell) associated to choice mechanism of gastropod shells by $P$. criniticornis.

\section{Material And Methods}

\section{Study area}

Located in Ubatuba city, Anchieta Island (233' S $45^{\circ} 05^{\prime} \mathrm{W}$ ) is the second largest island of the north coast of São Paulo State, with a total area of about 10 $\mathrm{km}^{2}$. This island has been affected by anthropogenic activities such as tourism and fishery exploitation until 1977, when it was declared an ecological reserve of São Paulo State (see Mantelatto and Garcia, 2002 for details).

\section{Shell occupation pattern}

The samples were obtained in the infralittoral rocky/ sandy area from January to December 1999. The hermit crabs were captured by two persons using SCUBA diving methods during $30 \mathrm{~min}$ over the same area of about $800 \mathrm{~m}^{2}$, at 9-10 $\mathrm{m}$ deep. After collection, the animals were frozen and transported to the laboratory where they were fixed in $80 \%$ alcohol for later analysis. Animals were removed from their shells, counted, measured (CSL $=$ Cephalothoracic Shield Length), and weighed (WW = Wet Weight). Shell species occupied by these individuals were identified according to Rios (1994) and the taxonomic nomenclature revised by a specialist, measured (SAW $=$ Shell Aperture Width and SAL $=$ Shell Aperture Length) and their wet weight (SWW) was determined. Shell internal volume (SIV) was measured by the method of Bertness (1981 b) modified by Mantelatto and Garcia (2000) by filling the empty shells with fine sand $\left(\varnothing=0.25 \mathrm{~mm}^{3}\right)$ with a pipette $\left(0.01 \mathrm{~mm}^{3}\right)$. The voucher crabs were deposited in the Crustacean Collection of the Biology Department (CCDB) of Faculty of Philosophy, Science and Letters at Ribeirão Preto (FFCLRP), University University of São Paulo (USP) (catalogue numbers: 
from CCDB 1476 to 1484 and 1741 to 1745 ).

The mean size of individuals of both sexes was compared by the Mann-Whitney test (Zar, 1996). The level of significance was 0.05 .

The relations between hermit crabs size and shell variables were determined by regression analysis and by correlation coefficients. The chi-square test $\left(\chi^{2}\right)$ was used to compare occupancy percentage of shell species between males, ovigerous females and nonovigerous females.

\section{Shell selection under laboratory conditions}

After the knowledge of the population profile (Mantelatto and Garcia, 2002; Mantelatto et al., 2007) and of the pattern of shell utilization by this population in the field, two types of laboratory experiments were performed: shell species and size preferences, which were conducted for the most occupied shell types in the field. For this, samples were additionally carried out during 2002 in the same area, following the above described method of collection.

Three replicates were conducted for each type of experiment, which occurred in a glass aquarium $(30 \times 30 \times 40 \mathrm{~cm})$ with flowing and oxygen-saturated seawater, where the animals chosen independently of sex or size $(\mathrm{N}=15)$ were placed naked with a large number of shells $(\mathrm{N}=150)$, during 72 hours (time previously established). After that, the hermit crabs were removed from the preferred shell, and both were measured as described above for shell occupation. Each animal was used only once in order to avoid any acquired behavior.

The shell species experiments were conducted in pair-wise fashion, i.e., the hermit crabs were placed in the aquarium with empty shells of two different species and similar size ( 75 of each species) than those found in the field. Shell size preference was tested separately for most occupied shell species using shells of the same species but of varied sizes. All experiments were performed according to method described by Garcia and Mantelatto (2001).

Shell species preference was estimated based on the frequency of which species was chosen by the individuals. The chi-square test $\left(\chi^{2}\right)$ was used to compare occupancy percentage of shell species. Morphometric relationships were established by regression analysis and Spearman correlation coefficients $\left(r_{s}\right)(p<0.05)$.
Shell fitness of hermit crabs collected in the field was assessed using a shell adequacy index (SAI) (Vance, 1972a). This index is defined as: SAI = suitable crab size/actual crab size, where SAI = 1 indicates that, in field, the animal occupies a shell of adequate size while $\mathrm{SAI} \neq 1$ indicates a crab in a shell larger $(\mathrm{SAI}>1)$ or smaller $(\mathrm{SAI}<1)$ than adequate size.

\section{ReSULTS}

\section{Shell occupation pattern}

In total, 992 individuals were collected, represented by 432 (43.55\%) males, 263 (26.51\%) non-ovigerous females, and 297 (29.94\%) ovigerous females. The mean size of population was $1.88 \pm 0.39 \mathrm{~mm} \mathrm{CSL}$, being females $(1.85 \pm 0.35 \mathrm{~mm}$ CSL $)$ significantly smaller than males $(1.92 \pm 0.31 \mathrm{~mm})$.

Pagurus criniticornis was found occupying 16 species of gastropod shells in the natural habitat (Tab. 1). Cerithium atratum (Born, 1778) was the most occupied shell (89.31\%), followed by Morula nodulosa (Adams, $1845)(4.73 \%)$. From the total (992) of shells collected, $2.42 \%$ were much damaged, and thus non identifiable.

Shell species occupation as a function of hermit crab size is illustrated (Fig. 1). The diversity of shells utilized decreased with the increase of individuals' size. Cerithium atratum was the most occupied shell in all size classes, except in the first class, where this corresponded to $12.5 \%$ of occupied shells. There was no difference between species of shell occupied by males, ovigerous females and non-ovigerous females.

The relationship between the shells and the hermit crabs was tested only to C. atratum and M. nodulosa, due to a low percentage of occupation for the other species of gastropod shells. The equations that best demonstrated this relationship were those that involved shell wet weight (SWW) and shell internal volume (SIV) (Tab. 2), mainly to the most occupied shell species, C. atratum.

\section{Experiments}

The experiments were conducted only for most the occupied species of shell, i.e., C. atratum and $M$. nodulosa.

\section{Shell species preference}

Forty-three animals were utilized ( 21 males, 14 nonovigerous females and 8 ovigerous females). Males 
Table 1. List of gastropod shells occupied by Pagurus criniticornis at Anchieta Island, São Paulo, Brazil.

\begin{tabular}{|c|c|c|c|c|c|c|c|c|}
\hline \multirow[t]{2}{*}{ Species } & \multirow[t]{2}{*}{ Total } & \multirow[t]{2}{*}{$\%$} & \multicolumn{2}{|c|}{ Males } & \multicolumn{2}{|c|}{ Non-ovigerous females } & \multicolumn{2}{|c|}{ Ovigerous females } \\
\hline & & & $\mathbf{N}$ & $\%$ & $\mathbf{N}$ & $\%$ & $\mathbf{N}$ & $\%$ \\
\hline Anachis lyrata (G.B. Sowerby I, 1832) & 3 & 0.30 & 1 & 0.23 & - & - & 2 & 0.67 \\
\hline Astralium latispina (Phillipi, 1844) & 1 & 0.10 & 1 & 0.23 & - & - & - & - \\
\hline Lithopoma phoebium (Roding, 1798) & 2 & 0.20 & 1 & 0.23 & 1 & 0.38 & - & - \\
\hline Cerithium atratum (Born, 1778) & 886 & 89.31 & 390 & 90.3 & 227 & 86.31 & 269 & 90.57 \\
\hline Siratus tenuivaricosus (Dautzenberg, 1927) & 1 & 0.10 & 1 & 0.23 & - & - & - & - \\
\hline Coralliophila aberrans (C.B. Adams, 1850) & 1 & 0.10 & 1 & 0.23 & - & - & - & - \\
\hline Costoanachis sertulariarum (d’Orbigny, 1839) & 2 & 0.20 & - & - & 1 & 0.38 & 1 & 0.33 \\
\hline Fusinus brasiliensis (Grabau, 1904) & 1 & 0.10 & 1 & 0.23 & - & - & - & - \\
\hline Leucozonia nassa (Gmelin, 1791) & 4 & 0.40 & 3 & 0.69 & 1 & 0.38 & - & - \\
\hline Modulus modulus (Linnaeus, C., 1758) & 9 & 1.28 & 3 & 0.69 & 4 & 1.52 & 2 & 0.67 \\
\hline Morula nodulosa (C.B. Adams, 1845) & 47 & 4.73 & 12 & 2.78 & 14 & 5.32 & 21 & 7.10 \\
\hline Muricopsis necocheana (Pilbry, 1900) & 1 & 0.10 & 1 & 0.23 & - & - & - & - \\
\hline Nassarius albus (Say, 1826) & 4 & 0.40 & 3 & 0.69 & 1 & 0.38 & - & - \\
\hline Gemophos auritulus (Link, 1807) & 2 & 0.20 & 1 & 0.69 & 1 & 0.38 & - & - \\
\hline Polinices lacteus (Guilding, 1834) & 2 & 0.20 & 2 & 0.46 & - & - & - & - \\
\hline Tegula viridula (Gmelin, 1791) & 2 & 0.20 & 1 & 0.69 & 1 & 0.38 & - & - \\
\hline No identification & 24 & 2.42 & 10 & 2.31 & 12 & 4.56 & 2 & 0.67 \\
\hline Total & 992 & & 432 & & 263 & 4.56 & 297 & \\
\hline
\end{tabular}

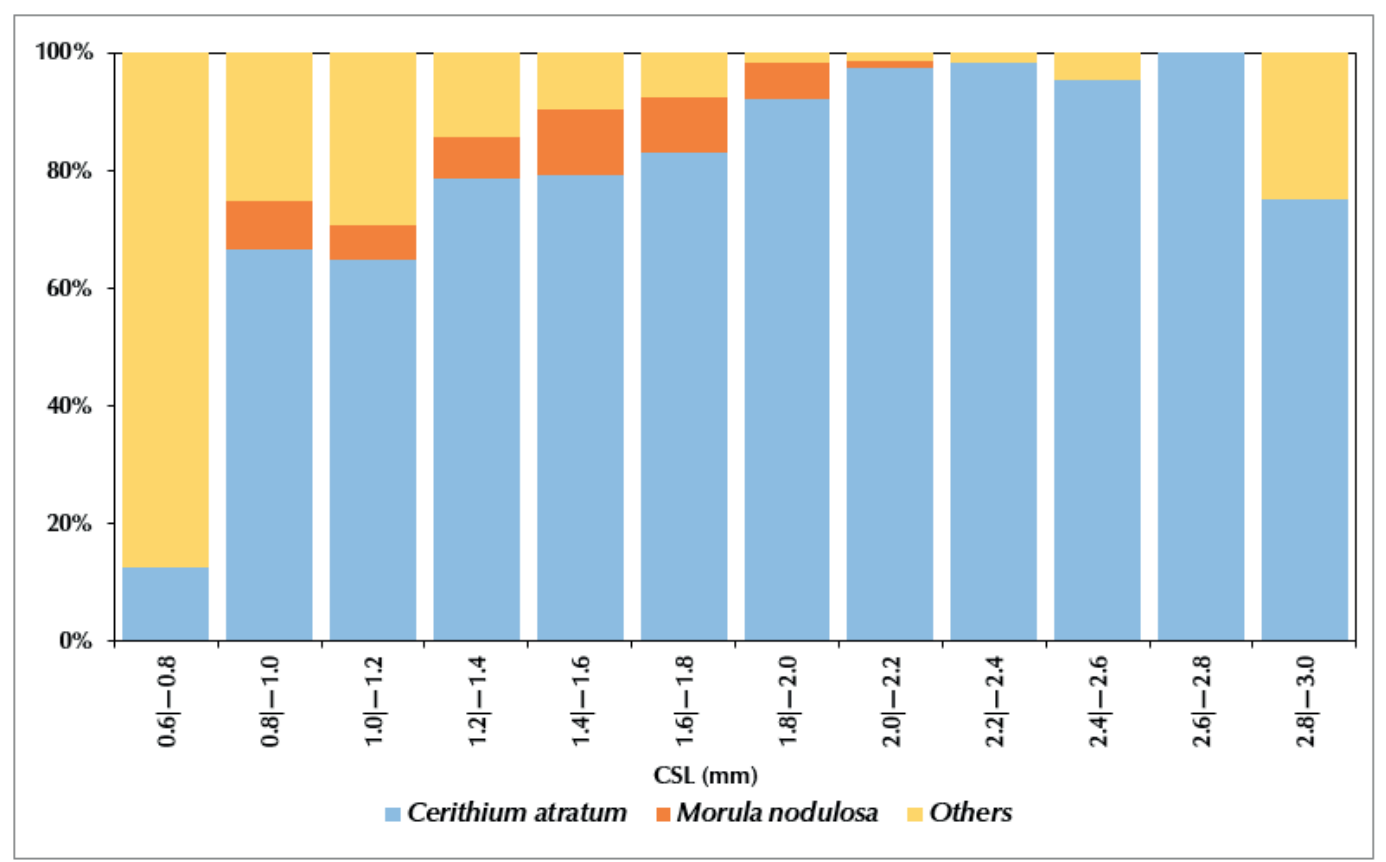

Figure 1. Pagurus criniticornis. Gastropod shell species occupation as a function of hermit crab size. (CSL = Cephalothoracic Shield Length).

$\left(\chi^{2}=17.19\right)$ and non-ovigerous females $\left(\chi^{2}=4.57\right)$ of Pagurus criniticornis, but not ovigerous females $\left(\chi^{2}=\right.$ $2.00)$, showed a significant $(\mathrm{p}<0.05)$ preference by C. atratum (Tab. 3).

Shell size preference

One hundred one animals were utilized (48 males, 32 non-ovigerous females and 21 ovigerous females). All relations between dimensions of hermit crabs and selected shells were significant $(\mathrm{p}<0.05)$, but $P$. criniticornis showed a preference strongly associated with SIV and SWW. The higher correlation coefficients demonstrated that $C$. atratum shells are most adequate to the various hermit crab sizes (Tab. 4). The pattern of shell choice of males, non-ovigerous females and ovigerous females was not different for both species of shells (Tabs. 5, 6). 
Table 2. Pagurus criniticornis. Regression equations for measures between hermit crab (CSL = Cephalothoracic Shield Length and $\mathrm{WW}=$ hermit crab Wet Weight) and the two most occupied species of shells (SAW = Shell Aperture Width; SAL = Shell Aperture Length; SWW = Shell Wet Weight; SIV = Shell Internal Volume). [ $\mathrm{N}=$ number of individuals; $\mathrm{r}_{\mathrm{s}}=$ Spearman correlation coefficient $]$.

\begin{tabular}{|c|c|c|c|c|c|}
\hline Species & Variables & $\mathbf{N}$ & $\mathbf{Y}=\mathbf{a} \mathbf{X}^{\mathbf{b}}$ & $\ln Y=\ln a+b \ln X$ & $\mathbf{r}_{\mathrm{s}}$ \\
\hline \multirow{8}{*}{ Cerithium atratum } & CSL x SAW & 886 & $\mathrm{SAW}=1.52 \mathrm{CSL}^{0.92}$ & $\ln \mathrm{SAW}=0.42+0.92 \ln \mathrm{CSL}$ & $0.62^{*}$ \\
\hline & CSL x SAL & 886 & $\mathrm{SAL}=0.02 \mathrm{CSL}^{1.35}$ & $\ln \mathrm{SAL}=0.70+1.35 \ln \mathrm{CSL}$ & $0.64^{*}$ \\
\hline & CSL x SWW & 886 & $\mathrm{SWW}=0.11 \mathrm{CSL}^{2.15}$ & $\ln S W W=-2.21+2.15 \ln \mathrm{CSL}$ & $0.65^{*}$ \\
\hline & CSL x SIV & 321 & $\mathrm{SIV}=0.02 \mathrm{CSL}^{2.25}$ & $\ln \mathrm{SIV}=-3.91+2.25 \ln \mathrm{CSL}$ & $0.71^{*}$ \\
\hline & WW x SAW & 886 & $\mathrm{SAW}=6.87 \mathrm{WW}^{0.27}$ & $\ln \mathrm{SAW}=1.93+0.27 \ln \mathrm{WW}$ & $0.68^{*}$ \\
\hline & WW x SAL & 886 & $\mathrm{SAL}=17.48 \mathrm{WW}^{0.38}$ & $\ln \mathrm{SAL}=2.86+0.38 \ln \mathrm{WW}$ & $0.67^{*}$ \\
\hline & WW x SWW & 886 & $\mathrm{SWW}=3.80 \mathrm{WW}^{0.62}$ & $\ln \mathrm{SWW}=1.33+0.62 \ln \mathrm{WW}$ & $0.71^{*}$ \\
\hline & WW x SIV & 321 & $\mathrm{SIV}=0.90 \mathrm{WW}^{0.63}$ & $\ln \mathrm{SIV}=-0.10+0.63 \ln \mathrm{WW}$ & $0.75^{*}$ \\
\hline \multirow{8}{*}{ Morula nodulosa } & CSL x SAW & 47 & $\mathrm{SAW}=1.37 \mathrm{CSL}^{0.59}$ & $\ln \mathrm{SAW}=0.30+0.59 \ln \mathrm{CSL}$ & $0.33^{*}$ \\
\hline & CSL x SAL & 47 & $\mathrm{SAL}=2.66 \mathrm{CSL}^{0.08}$ & $\ln \mathrm{SAL}=0.98+0.08 \ln \mathrm{CSL}$ & 0.28 \\
\hline & CSL x SWW & 47 & $\mathrm{SWW}=0.19 \mathrm{CSL}^{1.62}$ & $\ln S W W=-1.66+1.62 \ln C S L$ & $0.48^{*}$ \\
\hline & CSL x SIV & 28 & $\mathrm{SIV}=0.03 \mathrm{CSL}^{1.48}$ & $\ln \mathrm{SIV}=-3.50+1.48 \ln \mathrm{CSL}$ & $0.72^{*}$ \\
\hline & WW x SAW & 47 & $\mathrm{SAW}=3.56 \mathrm{WW}^{0.17}$ & $\ln S A W=1.27+0.17 \ln W W$ & $0.39^{*}$ \\
\hline & WW x SAL & 47 & $\mathrm{SAL}=10.98 \mathrm{WW}^{0.22}$ & $\ln S A L=2.39+0.22 \ln W W$ & 0.20 \\
\hline & WW x SWW & 47 & $\mathrm{SWW}=2.30 \mathrm{WW}^{0.44}$ & $\ln \mathrm{SWW}=0.83+0.44 \ln \mathrm{WW}$ & $0.51^{*}$ \\
\hline & WW x SIV & 21 & $\mathrm{SIV}=0.19 \mathrm{WW}^{0.27}$ & $\ln \mathrm{SIV}=-1.66+0.27 \ln \mathrm{WW}$ & $0.56^{*}$ \\
\hline
\end{tabular}

* Statistically significant, $\mathrm{p}<0.05$

Table 3. Pagurus criniticornis. Laboratory shell species preference observed among the two most occupied in the field.

\begin{tabular}{cccc}
\hline \multirow{2}{*}{ Sex category } & N & \multicolumn{2}{c}{ Selected shell species } \\
\cline { 3 - 4 } & & Cerithium atratum & Morula nodulosa \\
\hline Males & 21 & 20 & 01 \\
Non-ovigerous females & 14 & 11 & 03 \\
Ovigerous females & 8 & 06 & 02 \\
\hline
\end{tabular}

* Statistically significant; ns = not significant; $\mathrm{p}<0.05$

Table 4. Pagurus criniticornis. Regression analysis for each chosen shell size (CSL = Cephalothoracic Shield Length; WW = hermit crab Wet Weight; SAW = Shell Aperture Width; SWW = Shell Wet Weight; SIV = Shell Internal Volume; $\mathrm{N}=$ number of individuals; $r_{s}=$ Spearman correlation coefficient).

\begin{tabular}{|c|c|c|c|c|c|}
\hline Species & Variables & $\mathbf{N}$ & $\mathbf{Y}=\mathbf{a} \mathbf{X}^{\mathbf{b}}$ & $\ln Y=\ln a+b \ln X$ & $\mathbf{r}_{\mathrm{s}}$ \\
\hline \multirow{6}{*}{ Cerithium atratum } & CSL x SAW & 57 & $\mathrm{SAW}=1.79 \mathrm{CSL}^{0.72}$ & $\ln \mathrm{SAW}=0.58+0.72 \ln \mathrm{CSL}$ & $0.68^{*}$ \\
\hline & CSL x SWW & 57 & $S W W=0.08 \mathrm{CSL}^{2.35}$ & $\ln S W W=-2.52+2.35 \ln C S L$ & $0.84^{*}$ \\
\hline & CSL x SIV & 48 & $\mathrm{SIV}=0.03 \mathrm{CSL}^{2.07}$ & $\ln \mathrm{SIV}=-3.50+2.07 \ln \mathrm{CSL}$ & $0.77^{*}$ \\
\hline & WW x SAW & 57 & $\mathrm{SAW}=6.18 \mathrm{WW}^{0.24}$ & $\ln \mathrm{SAW}=1.82+0.24 \ln \mathrm{WW}$ & $0.71^{*}$ \\
\hline & WW x SWW & 57 & $\mathrm{SWW}=3.39 \mathrm{WW}^{0.70}$ & $\ln S W W=1.22+0.70 \ln W W$ & $0.82^{*}$ \\
\hline & WW x SIV & 48 & $\mathrm{SIV}=1.20 \mathrm{WW}^{0.69}$ & $\ln \mathrm{SIV}=0.18+0.69 \ln \mathrm{WW}$ & $0.80^{*}$ \\
\hline \multirow{6}{*}{ Morula nodulosa } & CSL x SAW & 44 & $\mathrm{SAW}=1.90 \mathrm{CSL}^{0.43}$ & $\ln \mathrm{SAW}=0.64+0.43 \ln \mathrm{CSL}$ & $0.33^{*}$ \\
\hline & CSL x SWW & 44 & $\mathrm{SWW}=0.10 \mathrm{CSL}^{2.50}$ & $\ln \mathrm{SWW}=-2.30+2.50 \ln \mathrm{CSL}$ & $0.62^{*}$ \\
\hline & CSL x SIV & 43 & $\mathrm{SIV}=0.02 \mathrm{CSL}^{2.07}$ & $\ln \mathrm{SIV}=-3.91+2.07 \ln \mathrm{CSL}$ & $0.65^{*}$ \\
\hline & WW x SAW & 44 & $\mathrm{SAW}=3.55 \mathrm{WW}^{0.11}$ & $\ln \mathrm{SAW}=1.26+0.11 \ln \mathrm{WW}$ & $0.30^{*}$ \\
\hline & WW x SWW & 44 & $\mathrm{SWW}=3.16 \mathrm{WW}^{0.96}$ & $\ln \mathrm{SWW}=1.15+0.06 \ln \mathrm{WW}$ & $0.51^{*}$ \\
\hline & WW x SIV & 43 & $\mathrm{SIV}=0.49 \mathrm{WW}^{0.51}$ & $\ln \mathrm{SIV}=-0.71+0.51 \ln \mathrm{WW}$ & $0.58^{*}$ \\
\hline
\end{tabular}

* Statistically significant, $\mathrm{p}<0.05$

\section{Shell Adequacy Index}

The mean SAI value found to $P$. criniticornis population was $1.13 \pm 0.25$. Shells occupied by hermit crabs in the field were larger than adequate size $(\mathrm{SAI}>1)$ in the most size classes; however, larger individuals showed a trend to occupy shells of adequate size $(\mathrm{SAI}=1)$.

There were no differences in this pattern observed between males, non-ovigerous females and ovigerous females (Fig. 2). For the two most occupied shell species tested the pattern of adequacy was similar, 
Table 5. Pagurus criniticornis. Regression equations for measures between hermit crab and the shells of Cerithium atratum chosen under laboratory conditions, according to sexes. (CSL = Cephalothoracic Shield Length; WW = hermit crab Wet Weight; SAW = Shell Aperture Width; SWW = Shell Wet Weight; SIV = Shell Internal Volume; $\mathrm{N}=$ number of individuals; $\mathrm{r}_{\mathrm{s}}=$ Spearman correlation coefficient).

\begin{tabular}{|c|c|c|c|c|c|}
\hline Sexes & Variables & $\mathbf{N}$ & $\mathbf{Y}=\mathbf{a} \mathbf{X}^{\mathbf{b}}$ & $\ln Y=\ln a+b \ln X$ & $\mathbf{r}_{\mathrm{s}}$ \\
\hline \multirow{6}{*}{ Males } & CSL x SAW & 27 & $\mathrm{SAW}=2.08 \mathrm{CSL}^{0.53}$ & $\ln \mathrm{SAW}=0.73+0.53 \ln \mathrm{CSL}$ & $0.56^{*}$ \\
\hline & CSL x SWW & 27 & $\mathrm{SWW}=0.11 \mathrm{CSL}^{1.86}$ & $\ln S W W=-2.20+1.86 \ln C S L$ & $0.82^{*}$ \\
\hline & CSL x SIV & 23 & $\mathrm{SIV}=0.06 \mathrm{CSL}^{1.30}$ & $\ln \mathrm{SIV}=-2.81+1.30 \ln \mathrm{CSL}$ & $0.62^{*}$ \\
\hline & WW x SAW & 27 & $\mathrm{SAW}=5.14 \mathrm{WW}^{0.18}$ & $\ln \mathrm{SAW}=1.64+0.18 \ln \mathrm{WW}$ & $0.67^{*}$ \\
\hline & WW x SWW & 27 & $S W W=1.89 W^{0.51}$ & $\ln S W W=0.64+0.51 \ln W W$ & $0.81^{*}$ \\
\hline & WW x SIV & 23 & $\mathrm{SIV}=0.53 \mathrm{WW}^{0.44}$ & $\ln S I V=-0.63+0.44 \ln W W$ & $0.73^{*}$ \\
\hline \multirow{6}{*}{ Non-ovigerous females } & CSL x SAW & 19 & $\mathrm{SAW}=1.52 \mathrm{CSL}^{0.92}$ & $\ln \mathrm{SAW}=0.42+0.92 \ln \mathrm{CSL}$ & $0.69^{*}$ \\
\hline & CSL $x$ SWW & 19 & $\mathrm{SWW}=0.06 \mathrm{CSL}^{2.63}$ & $\ln \mathrm{SWW}=-2.81+2.63 \ln \mathrm{CSL}$ & $0.73^{*}$ \\
\hline & CSL x SIV & 14 & $\mathrm{SIV}=0.02 \mathrm{CSL}^{2.72}$ & $\ln \mathrm{SIV}=-3.91+2.72 \ln \mathrm{CSL}$ & $0.81^{*}$ \\
\hline & WW x SAW & 19 & $\mathrm{SAW}=7.84 \mathrm{WW}^{0.31}$ & $\ln \mathrm{SAW}=2.05+0.31 \ln \mathrm{WW}$ & $0.75^{*}$ \\
\hline & WWx SWW & 19 & $\mathrm{SWW}=7.90 \mathrm{WW}^{0.33}$ & $\ln S W W=2.10+0.33 \ln W W$ & $0.82^{*}$ \\
\hline & WW x SIV & 14 & $\mathrm{SIV}=3.48 \mathrm{WW}^{1.03}$ & $\ln \mathrm{SIV}=1.23+1.03 \ln \mathrm{WW}$ & $0.91^{*}$ \\
\hline \multirow{6}{*}{ Ovigerous females } & CSL x SAW & 11 & $\mathrm{SAW}=1.68 \mathrm{CSL}^{0.22}$ & $\ln \mathrm{SAW}=0.52+0.22 \ln \mathrm{CSL}$ & $0.74^{*}$ \\
\hline & CSL x SWW & 11 & $\mathrm{SWW}=0.06 \mathrm{CSL}^{2.75}$ & $\ln S W W=-2.81+2.75 \ln C S L$ & $0.93^{*}$ \\
\hline & CSL x SIV & 11 & $\mathrm{SIV}=0.03 \mathrm{CSL}^{2.44}$ & $\ln \mathrm{SIV}=-3.50+2.44 \ln \mathrm{CSL}$ & $0.87^{*}$ \\
\hline & WW x SAW & 11 & $\mathrm{SAW}=6.90 \mathrm{WW}^{0.28}$ & $\ln S A W=1.93+0.28 \ln W W$ & $0.70^{*}$ \\
\hline & WW x SWW & 11 & $\mathrm{SWW}=7.40 \mathrm{WW}^{0.96}$ & $\ln S W W=2.06+0.96 \ln W W$ & $0.85^{*}$ \\
\hline & WW x SIV & 11 & $\mathrm{SIV}=2.02 \mathrm{WW}^{0.88}$ & $\ln \mathrm{SIV}=0.70+0.88 \ln \mathrm{WW}$ & $0.85^{*}$ \\
\hline
\end{tabular}

* Statistically significant, $\mathrm{p}<0.05$
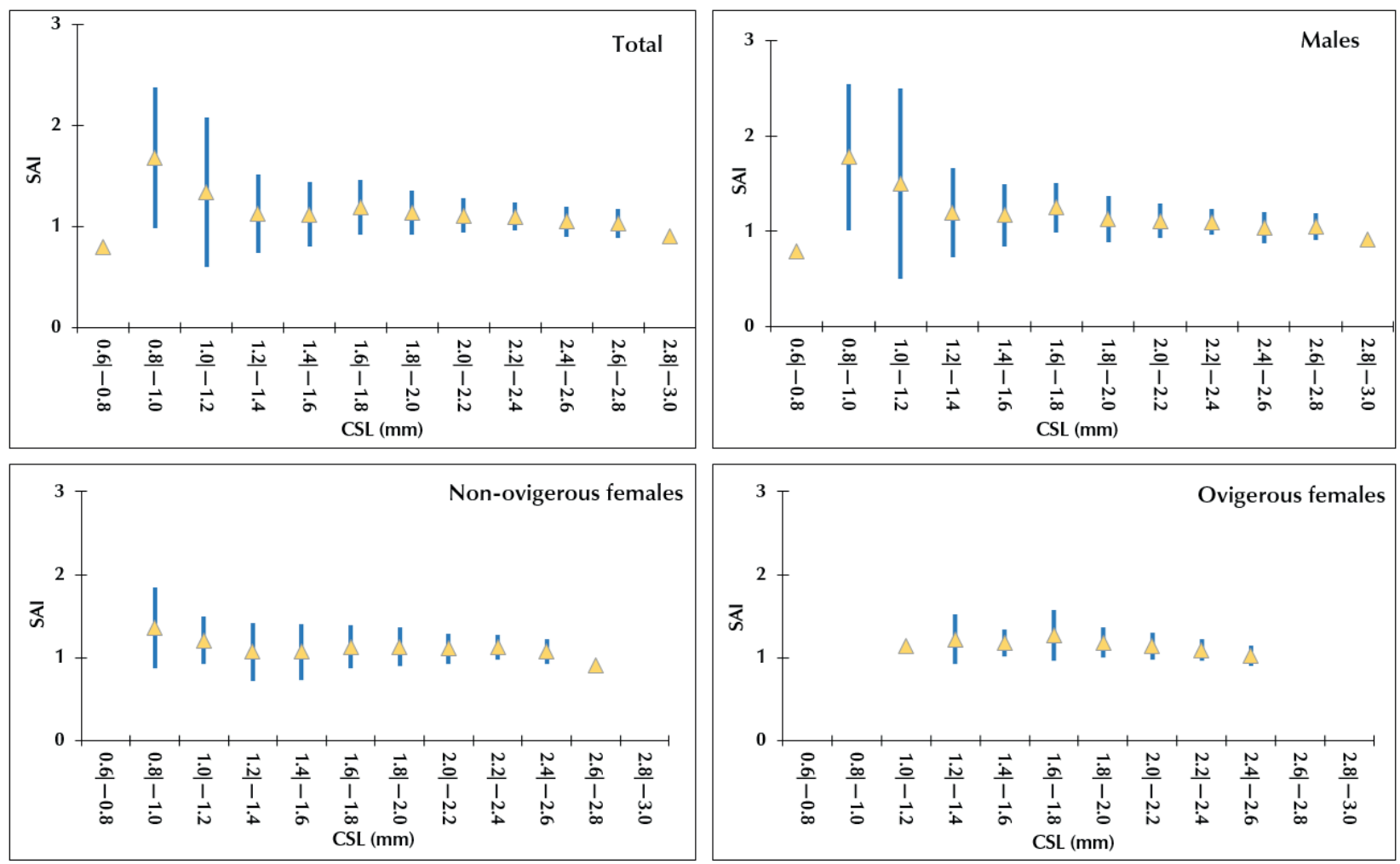

Figure 2. Pagurus criniticornis. Variation of the mean shell adequacy index (SAI) values for total of population, males, non-ovigerous females and ovigerous females. (Data are expressed as mean \pm SD) 
Table 6. Pagurus criniticornis. Regression equations for measures between hermit crab and the shells of Morula nodulosa chosen under laboratory conditions, according to sexes. (CSL = Cephalothoracic Shield Length; WW = hermit crab Wet Weight; SAW = Shell Aperture Width; SWW = Shell Wet Weight; SIV = Shell Internal Volume; N = number of individuals; rs = Spearman correlation coefficient).

\begin{tabular}{|c|c|c|c|c|c|}
\hline Sexes & Variables & $\mathbf{N}$ & $\mathbf{Y}=\mathbf{a} \mathbf{X}^{\mathbf{b}}$ & $\ln Y=\ln a+b \ln X$ & $\mathbf{r}_{\mathrm{s}}$ \\
\hline \multirow{6}{*}{ Males } & CSL x SAW & 21 & $\mathrm{SAW}=2.13 \mathrm{CSL}^{0.20}$ & $\ln \mathrm{SAW}=0.76+0.20 \ln \mathrm{CSL}$ & 0.20 \\
\hline & CSL x SWW & 21 & $\mathrm{SWW}=0.12 \mathrm{CSL}^{2.12}$ & $\ln S W W=-2.12+2.12 \ln C S L$ & $0.56^{*}$ \\
\hline & CSL x SIV & 20 & $\operatorname{SIV}=0.02 \mathrm{CSL}^{2.07}$ & $\ln \mathrm{SIV}=-3.91+2.07 \ln \mathrm{CSL}$ & $0.66^{*}$ \\
\hline & WW $\mathrm{x}$ SAW & 21 & $\mathrm{SAW}=3.33 \mathrm{WW}^{0.10}$ & $\ln \mathrm{SAW}=1.20+0.180 \ln \mathrm{WW}$ & 0.32 \\
\hline & WW x SWW & 21 & $S W W=4.07 W^{0.65}$ & $\ln S W W=1.40+0.65 \ln W W$ & $0.59^{*}$ \\
\hline & WW x SIV & 20 & $\mathrm{SIV}=0.78 \mathrm{WW}^{0.67}$ & $\ln S I V=-0.25+0.67 \ln W W$ & $0.72^{*}$ \\
\hline \multirow{6}{*}{ Non-ovigerous females } & CSL x SAW & 13 & $\mathrm{SAW}=1.70 \mathrm{CSL}^{0.62}$ & $\ln \mathrm{SAW}=0.53+0.62 \ln \mathrm{CSL}$ & 0.45 \\
\hline & CSL $x$ SWW & 13 & $S W W=0.05 C^{C S L} L^{3.54}$ & $\ln S W W=-2.99+3.54 \ln C S L$ & $0.73^{*}$ \\
\hline & CSL x SIV & 13 & $\mathrm{SIV}=0.21 \mathrm{CSL}^{2.20}$ & $\ln \mathrm{SIV}=-1.56+2.20 \ln \mathrm{CSL}$ & $0.63^{*}$ \\
\hline & WW x SAW & 13 & $\mathrm{SAW}=4.30 \mathrm{WW}^{0.15}$ & $\ln S A W=1.46+0.15 \ln W W$ & 0.48 \\
\hline & WW $x$ SWW & 13 & $\mathrm{SWW}=3.40 \mathrm{WW}^{0.56}$ & $\ln S W W=1.22+0.56 \ln W W$ & $0.56^{*}$ \\
\hline & WW x SIV & 13 & $\mathrm{SIV}=0.33 \mathrm{WW}^{0.38}$ & $\ln \mathrm{SIV}=-1.11+0.38 \ln \mathrm{WW}$ & 0.47 \\
\hline \multirow{6}{*}{ Ovigerous females } & CSL x SAW & 10 & $\mathrm{SAW}=0.97 \mathrm{CSL}^{1.48}$ & $\ln \mathrm{SAW}=-0.03+1.48 \ln \mathrm{CSL}$ & $0.68^{*}$ \\
\hline & CSL x SWW & 10 & $S W W=0.10 \mathrm{CSL}^{2.47}$ & $\ln S W W=-2.30+2.47 \ln C S L$ & 0.52 \\
\hline & CSL x SIV & 10 & $\mathrm{SIV}=0.02 \mathrm{CSL}^{2.12}$ & $\ln \mathrm{SIV}=-3.91+2.12 \ln \mathrm{CSL}$ & $0.68^{*}$ \\
\hline & WW x SAW & 10 & $\mathrm{SAW}=4.41 \mathrm{WW}^{0.18}$ & $\ln \mathrm{SAW}=1.48+0.18 \ln \mathrm{WW}$ & 0.22 \\
\hline & WW x SWW & 10 & $\mathrm{SWW}=1.35 \mathrm{WW}^{0.31}$ & $\ln S W W=0.30+0.31 \ln W W$ & 0.17 \\
\hline & WW x SIV & 10 & $\mathrm{SIV}=0.19 \mathrm{WW}^{0.81}$ & $\ln \mathrm{SIV}=-1.66+0.21 \ln \mathrm{WW}$ & 0.17 \\
\hline
\end{tabular}

* Significant correlation, $\mathrm{p}<0.05$
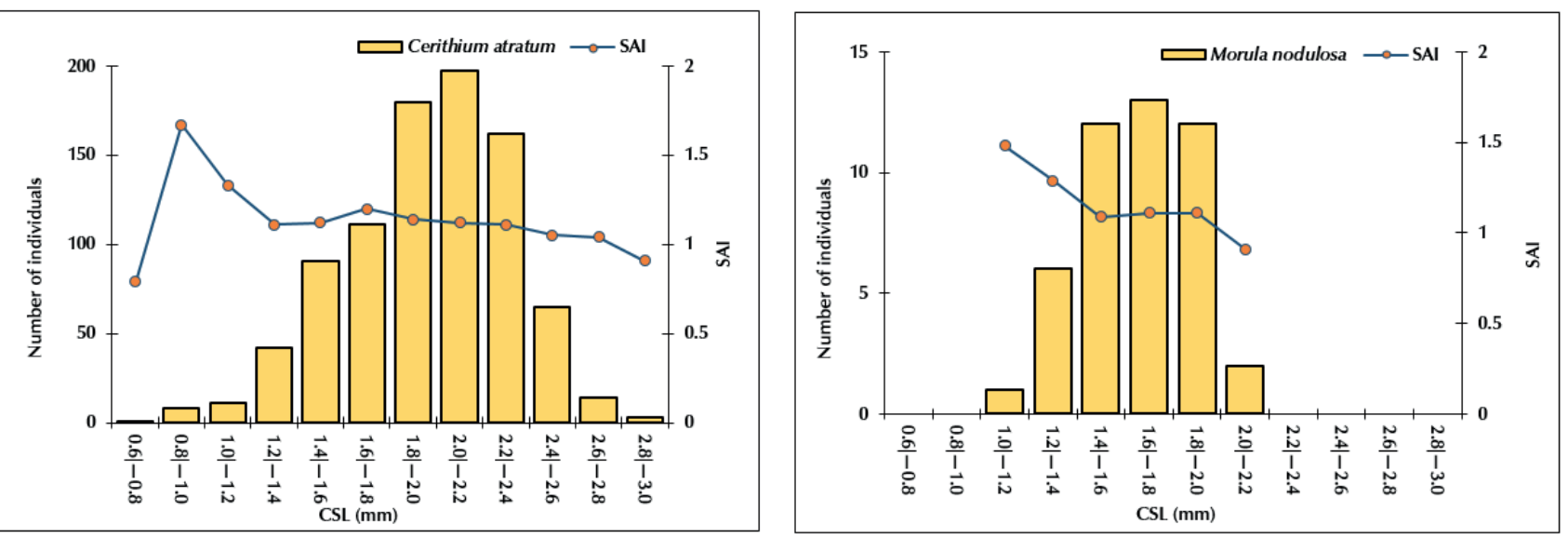

Figure 3. Pagurus criniticornis. Variation of the shell adequacy index (SAI) values for each most occupied shell species in relation to the size classes. (Data are expressed as mean $\pm \mathrm{SD}$ ).

i.e., $\mathrm{SAI}>1$ in the first size classes and this adequacy increased as the hermit crabs reached higher size classes (Fig. 3).

\section{Discussion}

Pagurus criniticornis was found occupying a large set of 16 gastropod shell species. Despite this diversity of occupied resource, there was not plasticity in shell type utilization, since C. atratum was the supranumerary occupied shell, with $89.31 \%$ of rate of occupation. Cerithium atratum was also one of the most frequently occupied shells by other small hermit crabs in this area, such as Paguristes tortugae (studied by Mantelatto and Dominciano, 2002), Pagurus brevidactylus (Mantelatto and Meireles, 2004), Calcinus tibicen (Mantelatto and Garcia, 2002) and Pseudopaguristes calliopsis (Biagi et al., 2006) in Anchieta Island area. This clear coexistence and the low percentage of damaged shells occupied by $P$. criniticornis demonstrated the good shell stock potential in this area, as accounted by Mantelatto and Garcia (2002), Meireles et al. (2003) and Mantelatto and Meireles (2004). This resource stock is one of the most 
important condition that allows the stable coexistence between species and do not cause a collapse in the use of resource and effects on population characteristics.

The pattern of shell utilization by hermit crabs is a result of a complex interaction among availability of shells (Reese, 1969; Bertness, 1980; Mantelatto and Garcia, 2000; Mantelatto and Dominciano, 2002; Mantelatto and Meireles, 2004) and selection of this resource, considering that hermit crabs can discriminate between different species and size of shells (Reese, 1962; 1963; Markham, 1968; Young, 1979; Hazlett, 1996; Mantelatto and Meireles, 2004).

Shell occupation pattern was more diverse in the initial size classes of $P$. criniticornis. According to Bollay (1964), individuals of smallest size tend to occur in most variety of small shells, since these are not heavily dependent on species of shells to survive and reproduce.

Patterns of shell utilization vary between hermit crab populations and are influenced by the type and size of shells available in the survey, the locality and hermit crabs' shell preference (Mantelatto and Garcia, 2002). Lively (1988) and Osorno et al. (1998) affirmed that hermit crabs prefer shells which the architecture maximizes their internal space. The present study corroborated this hypothesis, considering the good correlation values found between hermit crabs and $C$. atratum shells, mainly those related with weight and internal volume.

The availability of empty shells is a limiting factor to hermit crab populations, which is greatly influenced by the relative abundance of different live gastropods, their mortality rates (Bertness, 1980; Ohmori et al., 1995) and by inter/intraspecific competition (Reese, 1969). Meireles et al. (2003) studied the availability and diversity of local resources in Anchieta Island areas, where C. atratum was the most abundant shell, followed by Pisania auritula (Link, 1807), Astraea olfersii (Philippi, 1846) and M. nodulosa.

The shell occupation pattern of $P$. criniticornis showed similarity with availability of shells in the area; however, the rate of occupation of $C$. atratum and $M$. nodulosa by P. criniticornis was not proportional to the rate of availability found by Meireles et al. (2003), suggesting the preference for C. atratum as commented by Meireles et al. (2008). The experimental results confirmed the strong preference of individuals of this population for C. atratum shells, independently of sexes. This allows us to infer that this population's dynamics is directly related with C. atratum occupation.

The mean SAI value found for the population of P. criniticornis was 1.13 , which means that, in general, these animals utilize shells larger than the suitable size. However, value of shell adequacy index decreased with increase of crab size showing that the larger individuals were occupying relatively adequate shells. Meireles and Mantelatto (2005) observed similar pattern for the coexistent population of Pagurus brevidactylus from Anchieta Island.

The occupancy of larger shells than the adequate size by small individuals can be caused by an insufficient availability of small shells (Gherardi et al., 1994). According to Mantelatto and Meireles (2004), small shells represent a limited resource to the hermit crabs from Anchieta Island. Moreover, C. atratum was occupied by five of nine hermit crabs species that live in this area (Mantelatto and Garcia, 2002). In this case, the acquisition of shells larger than the adequate size is less strenuous than a continuous search for ideal sized shells.

In this way, the occurrence of individuals occupying larger shells than adequate ones is advantageous, since the interaction between specimens of the same population is more frequent than the probability of finding new and empty shells (Spight, 1977; 1985). These interactions allow changes that lead to better adequacy of individuals to their shells (Vance, 1972b) and consequently increase the potential of population growth. Thus, we are convinced that the pattern of adequacy observed in the studied population would be a satisfactory way to minimize the interspecific competition that it is exposed to.

When observed in an intertidal habitat, P. criniticornis showed abilities as explorer competitor and to acquire resources more rapidly than other species (Turra and Denadai, 2004). In this intertidal area, despite showing a preference for some species of gastropods, P. criniticornis does not seem to present such a rigorous pattern for selecting shells of higher quality or size, using mostly the shells that are available in the area, even if eroded or damaged (Turra and Denadai, 2004). Comparing these results with the obtained here, we can infer that differences are due to the resource availability and competition by coexistence species in the island 
assemblage, which make us to argue in favor of a future comparative study between both populations in order to elucidate this scenario.

The results obtained here indicate that the pattern of occupation found to P. criniticornis population is probably conducted by the availability of shells on the locality and by hermit crabs' choice of this resource, considering the relatively abundance of C. atratum on studied area and, additionally, the good adequacy of shell and hermit crab morphological characteristics, which is evidenced by high correlation coefficients, being, however, the second factor more influent under the determination of this pattern.

In recent studies, it was corroborated that in $P$. criniticornis population, there is a range of factors that influences shell exchange, and that some of them could represent trade-offs between immediate and long-term survival (Buranelli et al., 2015). In other words, these authors postulated that hermit crabs face different trade-offs related to the behavior of choice, selection, and exchange of shells, indicating that different factors may affect behavior, depending on many aspects as environmental features, sex, individual size, shell size, and shell quality.

\section{ACKNOWLEDGeMENTS}

The present study is part of the research project on hermit crab fauna from Anchieta Island and the major funding was provided by a grant to FLM by Fundação de Amparo à Pesquisa do Estado de São Paulo FAPESP (Proc. 1998/07454-5). This manuscript was also part of Master Dissertation project by FCRF, which is indebted to Conselho Nacional de Desenvolvimento Científico e Tecnológico - CNPq for a fellowship. $\mathrm{RB}$ and ALM are indebted to FAPESP (Procs. $1997 / 14245-0$ and 2000/02554-3; 1999/11679-5 and 2002/01646-7) for Master and Doctoral fellowships, respectively. FLM is grateful to $\mathrm{CNPq}$ for Research on-going fellowship (304968/2014-5). We thank Secretaria do Meio Ambiente do Estado de São Paulo, IBAMA and Parque Estadual da Ilha Anchieta for permission (Proc. 42358/98) and facilities during the sampling work. Special thanks are due to members of the Laboratory of Bioecology and Crustacean Systematics of FFCLRP/USP for help during the field and laboratory work, to the Programa de Pós
Graduação em Biologia Comparada - FFCLRP/USP for support and assistance, and to Helena MatthewsCascon (Universidade Federal do Ceará) for help with gastropod shell taxonomic revision. All experiments conducted in this study comply with current applicable state and federal laws. The authors are honored to recognize the many achievements of our friend and colleague, Nilton J. Hebling, by contributing to this commemorative issue.

\section{References}

Bertness, M.D. 1980. Shell preference and utilization patterns in littoral hermit crabs of the Bay of Panama. Journal of Experimental Marine Biology and Ecology, 48(1): 1-16.

Bertness, M.D. 1981a. Pattern and plasticity in tropical hermit crab growth and reproduction. American Naturalist, 117(5): 754-773.

Bertness, M.D. 1981b. The influence of shell-type on hermit crab growth rate and clutch size. Crustaceana, 40(2): 197-205.

Biagi, R.; Meireles, A.L. and Mantelatto, F.L. 2006. Bio-ecological aspects of the hermit crab Paguristes calliopsis (Crustacea, Diogenidae) from Anchieta Island, Brazil. Anais da Academia Brasileira de Ciências, 78(3): 451-462.

Bollay, M. 1964. Distribution and utilization of gastropod shells by the hermit crabs P. samuelis, P. granosimanus and P. hirsutiusculus at pacific groove, California. The Veliger, 6(suppl.): 71-76.

Buranelli, R.C.; Marcondes, A.T.P.; Carbonaro, F.A.; Miyazaki, M.J.; Pardo, L.M. and Mantelatto, F.L. 2015. Behavioral tradeoff on shell exchange and exploration of the white spotwrist hermit crab Pagurus criniticornis (Crustacea, Anomura, Paguridae). Crustacean Research, 44: 55-66.

Dominciano, L.C.C., Sant'anna, B.S. and Turra, A. 2009. Are the preference and selection patterns of hermit crabs for gastropod shells species or site-specific? Journal of Experimental Marine Biology and Ecology, 378(1): 15-21.

Garcia, R.B. and Mantelatto, F.L. 2001. Shell selection by the tropical hermit crab Calcinus tibicen (Herbst, 1791) (Anomura, Diogenidae) from Southern Brazil. Journal of Experimental Marine Biology and Ecology, 265: 1-14.

Gherardi, F.; Zatteri, F. and Vannini, M. 1994. Hermit crabs in a mangrove swamp: the structure of Clibanarius laevimanus clusters. Marine Biology, 121: 41-52.

Hazlett, B.A. 1996. Recent experience and the shell size preference of hermit crabs. Marine and Freshwater Behavior and Physiology, 28(3): 177-182.

Lively, C.M. 1988. A graphical model for shell-species selection by hermit crabs. Ecology, 69(4): 1233-1238.

Mantelatto, F.L. and Dominciano, L.C. 2002. Pattern of shell utilization by the hermit crab Paguristes tortugae (Diogenidae) from Anchieta Island, Southern Brazil. Scientia Marina, 66(3): 265-272. 
Mantelatto, F.L.; Faria, F.C.; Iossi, C.L. and Biagi, R. 2007. Population and reproductive features of the western Atlantic hermit crab Pagurus criniticornis (Anomura, Paguridae) from Anchieta Island, southeastern Brazil. Iheringia, Série Zoologia, 97(3): 1-7.

Mantelatto, F.L. and Garcia, R.B. 2000. Shell utilization pattern of the hermit crab Calcinus tibicen (Diogenidae) from southern Brazil. Journal of Crustacean Biology, 20(3): 460-467.

Mantelatto, F.L. and Garcia, R.B. 2002. Hermit crab fauna from the infralittoral area of Anchieta Island (Ubatuba, Brazil). p. 137-145. In: E.E. Briones and F. Alvarez (eds), Modern Approaches to the Studies of Crustacean. New York, Kluwer Academic/Plenum Publishers.

Mantelatto, F.L. and Meireles, A.L. 2004. The importance of shell occupation and shell availability clustering in the hermit crab Pagurus brevidactylus (Stimpson, 1859) (Paguridae) population from the Southern Atlantic. Bulletin of Marine Science, 75(1): 27-35.

Markham, J. 1968. Note on the growth patterns and shell utilization of hermit crab Pagurus bernhardus (L.). Ophelia, 5: 189-205.

Meireles, A.L.; Biagi, R. and Mantelatto, F.L. 2003. Gastropod shell availability as a potential resource for the hermit crab infralittoral fauna of Anchieta Island (SP), Brazil. Nauplius, 11(2): 99-105.

Meireles, A.L.; Biagi, R. and Mantelatto, F.L. 2008. Influence of prior experience on shell selection by the white spotwrist hermit crab Pagurus criniticornis (Crustacea: Paguridae). Hydrobiologia, 605: 259-263.

Meireles, A.L.; Biagi, R.; Fransozo, A. and Mantelatto, F.L. 2012. Os Ermitões (Crustacea, Anomura). P. 479-488. In: A.C.Z. Amaral \& S.A.H. Nallin (orgs), Biodiversidade e ecossistemas bentônicos marinhos do Litoral Norte de São Paulo - Sudeste do Brasil. Campinas, SP, IB/UNICAMP. E-book - ISBN:97885-85783-24-2 http://www.bibliotecadigital.unicamp.br/ document $/$ ?code $=000812694 \&$ opt $=1$

Meireles, A.L. and Mantelatto, F.L. 2005. Shell use by Pagurus brevidactylus (Anomura: Paguridae): a comparison between laboratory and field conditions. Acta Zoologica Sinica, 51 (5): 813-820.

Melo, G.A.S. 1999. Manual de Identificação dos Crustacea Decapoda do Litoral Brasileiro: Anomura, Thalassinidea,
Palinuridea, Astacidea. São Paulo, Editora Plêiade, 551p.

Ohmori, H.; Wada, S.; Goshima, S. and Nakao, S. 1995. Effects of body size and shell availability on the shell utilization pattern of the hermit crab Pagurus filholi (Anomura: Paguridae). Crustacean Research, 24: 85-92.

Osorno, J.L.; Fernández-Casillas, L. and Rodríguez-Juárez, C. 1998. Are hermit crabs looking for light and large shells?: evidence from natural and field induced shell exchanges. Journal of Experimental Marine Biology and Ecology, 222(1-2): 163-173.

Reese, E.S. 1962. Shell selection behaviour of hermit crabs. Animal Behaviour, 10(3-4): 347-360.

Reese, E.S. 1963. The behavioral mechanisms underlying shell selection by hermit crabs. Behaviour, 21: 78-126.

Reese, E.S. 1969. Behavioral adaptations of intertidal hermit crabs. American Zoologist, 9(2): 343-355.

Rios, E.C. 1994. Seashells of Brazil. Rio Grande, Fundação Cidade do Rio Grande, Instituto Acqua, Museu Oceanográfico de Rio Grande, Universidade de Rio Grande. 2a ED., 368p + 113pl.

Spight, T.M. 1977. Availability and use of shells by intertidal hermit crabs. The Biological Bulletin, 152(1): 120-133.

Spight, T.M. 1985. Why small hermit crabs have large shells. Research on Population Ecology, 27(1): 39-54.

Turra, A. and Denadai, M.R. 2004. Interference and exploitation components in interespecific competition between sympatric intertidal hermit crabs. Journal of Experimental Marine Biology and Ecology, 310(2): 183-193.

Vance, R.R. 1972a. The role of shell adequacy in behavioral interactions involving hermit crabs. Ecology, 53(6), 10751083.

Vance, R.R. 1972b. Competition and mechanism of coexistence of three sympatric species of intertidal hermit crabs. Ecology, 53(6): 1062-1074.

Young, A.M. 1979. Osmoregulation in three hermit crab species, Clibanarius vittatus (Bosc), Pagurus longicarpus Say and $P$. pollicaris Say (Crustacea: Decapoda; Anomura). Comparative Biochemistry and Physiology, 63A(3): 377-382.

Zar, J.H. 1996. Biostatiscal analysis. New Jersey, Prentice-Hall, $907 \mathrm{p}$. 